\title{
A Possibility to Obtain Equalities for the Critical Indices by Thermodynamic Way.
}

L. De Cesare

Osservatorio Astronomico - Caporlimonte (Napoli)

(Lett. Nuovo Cimento, 4, 251 (1972))

1) On p. 252, line 2 plase read

... of stable equilibrium.

2) The ref. $\left({ }^{3}\right)$ should read

R. B. Griffiths and J. WiIEeler: Phys. Rev. A, 2, 1047 (1970).

3) Line 7 of Table I should road

$\alpha_{\boldsymbol{H}}(T) \simeq A \cdot \beta\left(T_{e}-T\right)^{\beta-\mathbf{1}}$.

4) On p. 253, eq. (12) should read

$\cdots \gamma_{S}^{\prime} \leqslant \gamma_{T}^{\prime}=\gamma^{\prime}$.

5) Line 2 of note $\left(^{*}\right)$ p. 253 should read $\alpha^{\prime}+\gamma^{\prime}+2(\beta-1)<0$.

6) On p. 254 eq. (16) should read $\ldots\left(T \rightarrow T_{c}^{-}, H=0\right)$. 